\title{
Perianal Abscess with Fistula in Ano Complicating as Basal Cell Carcinoma- A Rare Presentation
}

\author{
Ramesh kumar A ${ }^{1 *}$, Bishal Mohanty $\mathrm{B}^{1}$ and Arun $\mathrm{P}^{2}$ \\ ${ }^{1}$ Registrar Surgery, Indraprastha Apollo Hospital, New Delhi, India \\ ${ }^{2}$ Department of Minimal Access Surgery, Indraprastha Apollo Hospital, India
}

*Corresponding author: Ramesh Kumar Aggarwal, DNB, Registrar Surgery,

\section{Case Report \\ Volume 2 Issue 2}

Received Date: February 12, 2018

Published Date: March 06, 2018

Indraprastha Apollo Hospital, New Delhi, India, Tel: 9958799558; Email: draggarwalramesh@gmail.cm

\section{Abstract}

Introduction: Fistula in ano usually presents with pus discharge Perianal region, sometimes with mucus discharge. Malignant transformation in fistula in Ano very rare presentation1, and to assess preoperatively is almost next to impossible, it requires a high index of clinical suspicion and proper workup.

Case Report: We present a very rare case of elderly female who initially presented with pus discharge and pain in Perianal region for 2-3 months, on through clinical examination and investigation (including MRI Fistulogram) done, a diagnosis of Perianal abscess and a high fistula in Ano was made. Fistulous tract which was sent for histopathological examination turned out as Nodular type of Basal Cell Carcinoma

Conclusion: Fistula in Ano is a benign disease usually present with pus discharge, with very rarely as malignant changes, a high index of suspicion should be kept if presenting in old age, with long standing history or with recurrent history or with history of mucus discharge or with some associated mass.

Keywords: Fistula-in Ano; BCC (Basal Cell Carcinoma); Fistulectomy

\section{Introduction}

Fistula in Ano usually presents with pus discharge Perianal region, sometimes with mucus discharge. Malignant transformation in fistula in Ano very rare presentation [1], and to assess preoperatively is almost next to impossible, it requires a high index of clinical suspicion and proper workup. Particularly if patient is in old age and if presenting with an mass or mucus discharge, one should suspect any hidden malignancy, and can think of preoperative biopsy of external opening or the fistulous tract. Although rare but squamous cell carcinoma and adenocarcinoma are the two common types which are reported in the literature. After studying more than 150-200 cases (PubMed) of fistula in Ano with carcinoma, we found only 1-2 cases reported, fistula in Ano with basal cell carcinoma.

\section{Case Report}

We present a very rare case of elderly female 73yr old, resident of Jangpura, new delhi(India) who initially presented with pus discharge and pain in Perianal region for 2-3 months, on through clinical examination and 


\section{International Journal of Surgery \& Surgical Techniques}

investigation (including MRI Fistulogram) done which showed transsphincteric fistula and collection posterior to anal canal/rectum secondary fistulous tract anteriorly with surrounding edematous changes ending blinding in supralevator region inferior to the pubic symphysis, a diagnosis of Perianal abscess and a high fistula in ano was made (Figures 1-3). Intraoperative sigmoidoscopy was also done which revealed the same, nothing abnormal than the mentioned. Under general anaesthesia Fistulectomy using methylene blue dye and drainage of abscess was done. Post operative period was uneventful. Fistulous tract which was sent for histopathological examination turned out as Nodular type of Basal Cell Carcinoma invading up to deep dermis (pT1NxMx), lymphnode and perineural invasion was not seen, deep resected margin \& lateral margin was involved by tumor (Figures 4,5). Patient was called again and wide excision of tumor bed was done with an curative intent.

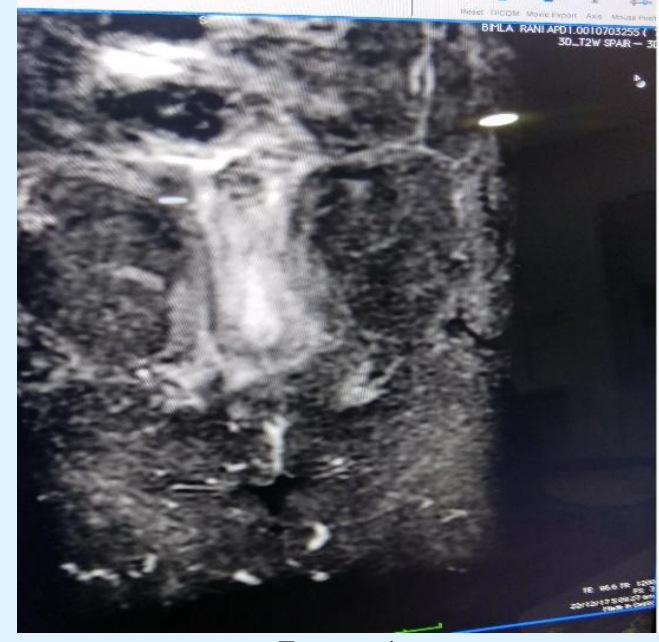

Figure 1

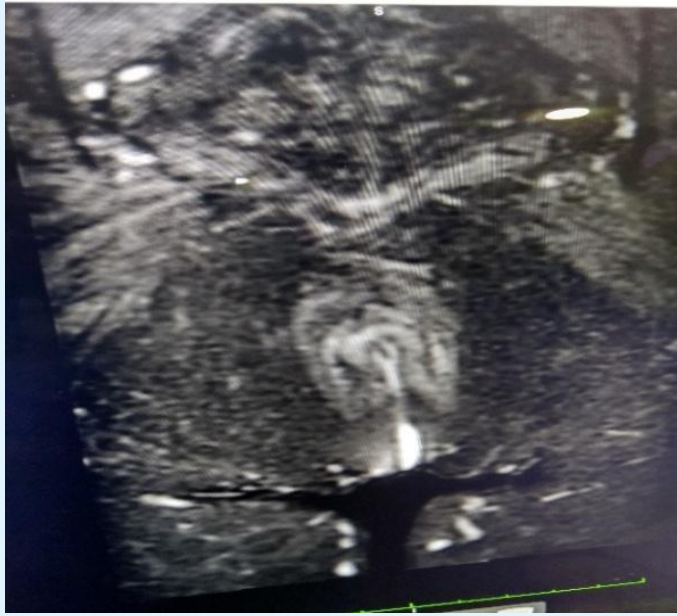

Figure 2

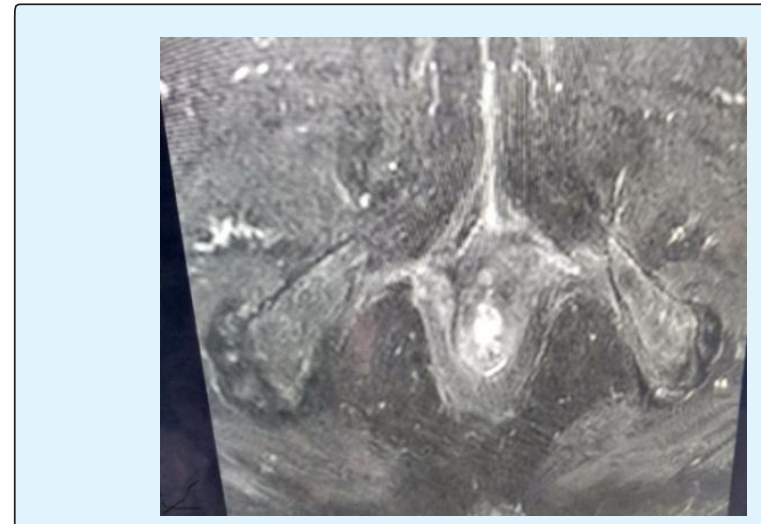

Figure 3

Figures 1-3: Figures 1-3 MRI Fistulogram-showing high fistula in ano with horse shoe shaped collection but with no features suggestive of malignancy.

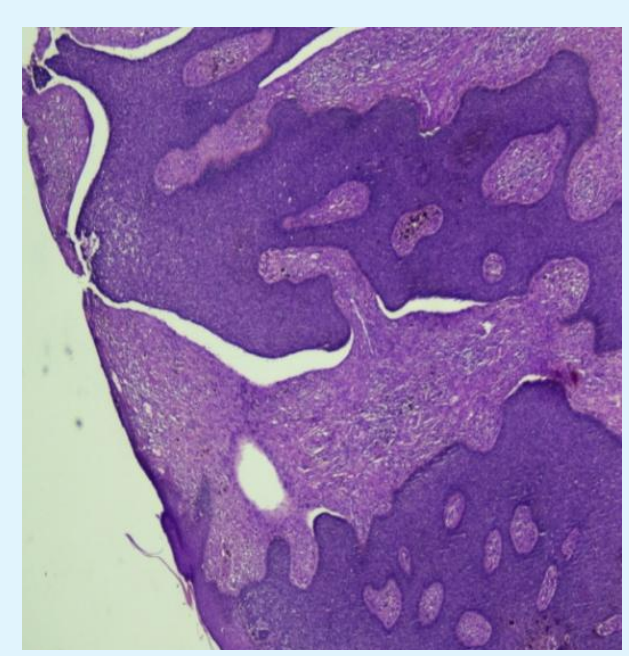

Figure 4

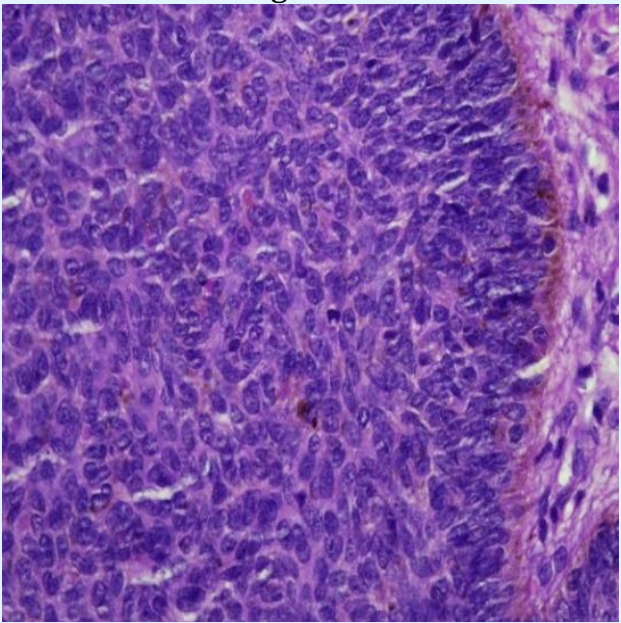

Figure 5

Figures 4 \& 5: Figures 4 \& 5 showing histopathology features of tumor as basal cell carcinoma. 


\section{International Journal of Surgery \& Surgical Techniques}

\section{Discussion}

Fistula in ano usually present with either the pus discharge or blood discharge in perianal region, very rarely it can present with an underlying mass or with mucus discharge, although this condition is very rare but in such condition one should suspect any association with malignancy. Fistula in ano is a benign disease itself can complicate from perianal abscess or it could be a manifestation of inflammatory bowel disease(crohn's disease) or due to tuberculosis(multiple fistula in perianal region).Due to rarity of this condition it is very hard to diagnose preoperatively or to assume weather the fistula is a presentation of malignancy or malignancy is a complication of long standing fistula, as some rectal cancer may present as fistula in ano, or some inflammatory bowel disease also present with fistula in ano. Rossner, et al. [2] in his study established three essential criteria to confirm that malignant transformation has occurred within fistula.

a) The fistula must be present for 10 years to exclude the possibility that the malignancy occurred before the fistula;

b) There should not be tumour within the mucosa of the rectum or anal canal unless there is definite evidence that this is metastatic tumour;

c) The Fistulous opening within the anal canal or rectum should not contain malignant tissue.

In our case except the chronicity fulfill the rossner's criteria. Difficulty in preoperative diagnosis is because

a) Due to very slow progress and onset of the tumour

b) In most of the cases in early stage tumour is asymptomatic, symptoms appear too late till then it is advance in stage.

c) Because the symptoms of fistula in Ano itself mask symptoms of malignancy if any.

Very few cases in literature are reported, who shows malignant transformation of fistulous tract, in most of them they are either adenocarcinoma, colloid carcinoma or squamous cell carcinoma, till now as per best of our knowledge, after going through the pub med literature only 1-2 cases of fistula in ano which turned out as Basal cell carcinoma are reported. GH Welch, et al. [3] in their study reported a suprasphincteric fistula in Ano with perianal abscess in elderly patient who had a history of multiple drainage and surgery, on Fistulogram and preoperative assessment biopsy of thickened mucosa was done which showed mucinous adenocarcinoma, managed with abdominoperineal resection. Most of the earlier reported cases are mucinous adenocarcinoma of low grade malignancy (44\%) [4]. In another study Raghavaiah $\mathrm{NV}$ reported a case of colloid carcinoma which developed in a long standing fistula in ano [5]. Takashima S, et al. in their study reported a case of long standing fistula with pus \& mucus discharge, preoperative cytology of discharge and biopsy of external opening was done which showed mucus adenocarcinoma, ultimately managed with abdomino-perineal resection with lymphnode dissection, they recommended that malignancy should be suspected in a long standing fistula history particularly those with mucus discharge[6]. Cirocchi R, et al. while reporting a case of recurrent fistula in an old aged patient who finally came out as mucinous adenocarcinoma, also recommended that preoperative evaluation, biopsy or cytology of recurrent abscess and fistulous tract should be done[7]. In all these cases there is characterstic long standing fistulous tract history and a preoperative suspicion was made on the basis of clinical history, a biopsy or cytology was done preoperatively which showed carcinoma, not like in our case, a very short history with no symptoms suggestive of malignancy, even on MRI Fistulogram no sign seen suggestive of malignancy. Basal cell carcinoma in fistulous tract came out as an surpriseelement which was not expected at al. Kulaylat MN, et al. also reported a similar case of fistula in ano, where in curettage specimen of fistulous tract they found basal cell carcinoma [8], like in our case where in biopsy of fistulous tract we found basal cell carcinoma, with no preoperative suspicion of malignancy. Manheim $\mathrm{SD}$, et al. also reported a case of basal cell carcinoma occurring in fistula in ano [9].

\section{Conclusion}

Fistula in ano is a benign disease usually present with pus discharge, with very rarely as malignant changes, a high index of suspicion should be kept if presenting in old age, with long standing history or with recurrent history or with history of mucus discharge or with some associated mass. A preoperative thorough investigation should be done including biopsy of the fistulous tract, cytology of recurrent abscess if needed and a proper clinical(including perrectal and proctoscopy examination , and every excised fistulous tract should be sent for histopathological examination, as though its rare but it can turn out as malignancy, and that should be managed accordingly by multidisciplinary approach.

\section{References}

1. Kyzer S, Bayer I, Turani H, Chaimoff C (1985) Verrucous squamous carcinoma as complication of recurrent multiple perianal fistulae. Colo-proctology 7: 104- 106. 


\section{International Journal of Surgery \& Surgical Techniques}

2. Rossner C (1934) Relation of fistula-in-ano to cancer of the anal canal. Trans Am Proc Soc pp: 65-70.

3. Welch GH, Finlay IG (1987) Neoplastic transformation in longstanding fistula-in-ano. Postgraduate Medical Journal 63(740): 503-504.

4. Kline RJ, Spencer RJ, Harrson EG (1964) Carcinoma associated with fistula-in-ano. Arch Surg 89(6): 989994.

5. Raghavaiah NV (1975) Colloid carcinoma arising in an anal fistula: case report with a brief review of the literature. Aust N Z J Surg 45(1): 97-99.
6. Takashima S, Fukunaga J, Kushibiki K, Kinami Y (1987) A case of carcinoma arising from an anal fistula. Gan No Rinsho 33(11): 1391-1396.

7. Cirocchi R, Covarelli P, Gullà N, Fabbri B, Bisacci R, et al. (1999) Adenocarcinoma arising from a recurrent fistula-in-ano. Ann Ital Chir 70(5): 771-774.

8. Kulaylat MN, Doerr RJ, Karamanoukian H, Barrios G (1996) Basal cell carcinoma arising in a fistula-in-ano. Am Surg 62(12): 1000-1002.

9. Manheim SD, Alexander RM (1955) Basal cell carcinoma occurring in a fistula-in ano. Am J Surg. 90(3): 522-525.

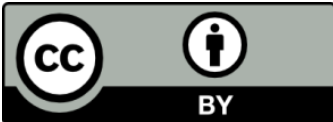

